\title{
Biostratigraphie et datations de la fin des temps glaciaires. Nouvelles visites des faunes de quelques gisements du Grand Sud-ouest de la France
}

Biostratigraphy and chronometric dates at the end of glacial times. A new assessment of the fauna from several sites from Southwestern France

\section{Françoise Delpech}

\section{OpenEdition Journals}

Édition électronique

URL : http://journals.openedition.org/paleo/5228

DOI : $10.4000 /$ paleo.5228

ISSN : 2101-0420

Éditeur

SAMRA

Édition imprimée

Date de publication : 1 septembre 2020

Pagination : 92-106

ISSN : 1145-3370

Référence électronique

Françoise Delpech, «Biostratigraphie et datations de la fin des temps glaciaires. Nouvelles visites des faunes de quelques gisements du Grand Sud-ouest de la France », PALEO [En ligne], 30-2 | 2020, mis en ligne le 30 septembre 2020, consulté le 10 décembre 2020. URL : http://journals.openedition.org/ paleo/5228; DOI : https://doi.org/10.4000/paleo.5228

\section{c) (i)}

PALEO est mis à disposition selon les termes de la licence Creative Commons Attribution - Pas d'Utilisation Commerciale - Pas de Modification 4.0 International. 
Avec l'augmentation de la précision et de la puissance des techniques et méthodes de recherche sur les événements du passé, les renseignements concernant la succession des strates et la date de leur formation se multiplient et sont de plus en plus précis. L'utilisation de ces renseignements conduit à proposer des relations temporelles nouvelles entre divers événements touchant les domaines environnemental et culturel, relations qui peuvent soit préciser, soit remettre en cause celles établies précédemment.

Nous appuyant sur des données concernant les grands mammifères ongulés, nous revisiterons le gisement de Pont-d'Ambon (Bourdeilles, Dordogne) et sept autres gisements de la fin des temps glaciaires. Par la prise en compte conjointe des datations et de la biostratigraphie, la disparition de la grande faune pléistocène du grand Sud-ouest de la France paraît être l'aboutissement d'un processus d'une durée quasi millénaire ; la période de sa mise en Françoise Delpecha

a. Chercheur bénévole PACEA, Université de Bordeaux, Allée Geoffroy SaintHilaire, CS 50023, FR-33615 Talence cedex - francoise67.delpech@orange.fr

PALEO 30 | t. 2

JUILLET 2020

PAGES 92 À 106

MOTS-CLÉS Biozonation, chronozonation, fin du Pléistocène, grands mammifères, Canis. 
Biostratigraphy and chronometric dates at the end of glacial times. A new assessment of the fauna from several sites from Southwestern France.

Increases in the precision and power of our research techniques and methods have led to a parallel increase in our understanding of the nature and chronology of stratigraphic succession in archaeological contexts. These increases allow a new assessment of the temporal relationships among diverse environmental and cultural events, in turn allowing us to understand those events with greater precision, and, in some cases, to question what we once though we knew.

Here, we revisit the site of Pont d'Ambon (Bourdeilles, Dordogne), and seven other sites of late glacial age, with a focus on large ungulates. A conjoined analysis of chronometric dates and stratigraphy shows that the disappearance of the late Pleistocene mammal fauna of Southwestern France was the result of a process that lasted about a millennium. Although this process varied across taxa, the end result was the same for all. In places marked by the expansion of deciduous forest, ungulates occupying open steppe environments (i.e., reindeer and saiga) and those occupying forested landscapes (i.e., red deer) decreased in size. The same size decrease impacted carnivores, including cave lions, which became smaller than the modern lion. The same thing may have been true for the wolf, suggesting caution for the assumption that small, wolflike members of the genus Canis from this context were dogs.

KEY-WORDS Biozonation, chronozonation, late Pleistocene, big mammals, Canis.

\section{INTRODUCTION}

J'ai longtemps eu l'intention d'écrire un livre sur la biostratigraphie des faunes du Pléistocène supérieur et ses implications chronologiques pour deux principales raisons :

1) les quelques articles dans lesquels j'en avais traitées (cf. bibliographie dans Delpech 2012) affichent des hypothèses qu'il est difficile de lier entre elles;

2) pour les questions de chronologie, chacun a aujourd'hui à sa disposition de nombreuses dates qui, traitées mathématiquement, situent précisément dans le temps l'arrivée, le développement et (ou) la disparition de tout événement ce qui a tendance à faire oublier les apports en la matière de la biostratigraphie. C'est ce à quoi contribuera sans doute un travail qui vient de paraître et qui fera, à n'en pas douter, autorité (Banks et al. 2019). Le livre que j'envisageais ne verra pas le jour. Cependant, la tenue du colloque : "L'Aquitaine à la fin des temps glaciaires» organisé par Aline Averbouh, Peggy BonnetJacquement et Jean-Jacques Cleyet-Merle, en hommage à Guy Célérier, le directeur des fouilles du gisement de Pontd'Ambon, m'a conduite à revoir et réunir données, anecdotes, réflexions et «regrets de n'avoir fait» concernant les faunes si particulières de ce gisement (Delpech 2018). Au cours de son écriture, il m'est apparu, une nouvelle fois, que la prise en compte de la biostratigraphie ne peut, en Archéologie préhistorique, être reléguée à un rang mineur ; avec la paléozoologie et la paléoécologie, elle se place au contraire au premier rang pour la connaissance des environnements du passé et en très bonne place pour les questions de chronologie. C'est donc sous cet angle de vue que nous tentons aujourd'hui de percevoir la fin des temps glaciaires dans un grand Sud-ouest de la France à partir des faunes de 8 gisements dont le Pont d'Ambon qui a servi de catalyseur.

\section{1 | BIOSTRATIGRAPHIE ET DATATIONS}

La biostratigraphie et les datations ouvrent deux voies de recherche qui apportent des informations, la première sur la chronologie relative des strates, la seconde sur l'ancienneté et l'homogénéité de chacune d'elles. Ces dernières années, la chronologie numérique semble avoir pris le pas sur les chronologies fondées sur la stratigraphie au point de faire presque oublier que c'est, avant tout, de l'ordonnancement des strates que l'on tire des informations sur la suite des événements de la Préhistoire. Ces datations ont largement précisé, voire revu, les estimations d'âge et de durée des événements qui étaient admises avant qu'elles n'entrent en scène. Les dernières grandes avancées viennent du fait que l'on peut maintenant situer ces événements relativement à des courbes de plus en plus nombreuses qui retracent avec finesse des changements climatiques et environnementaux enregistrés sous diverses formes dans les archives lacustres, océaniques et glaciaires. Ainsi, un mince événement enregistré en un point isolé du continent et daté peut être situé relativement à ces références à valeur globale. Par exemple, à partir d'un seul vestige animal découvert dans un gisement du Périgord, on peut non seulement dire à quel moment l'animal était présent dans l'environnement et en déduire à quel moment s'est formée la couche (ou le niveau) qui a fossilisé le vestige mais aussi la (le) corréler avec un Stadiaire ou un 
Interstadiaire Groenlandais, à un moment repaire de démantèlement de la banquise (événement d'Heinrich), voire aussi, le cas échéant, à une phase de la fin des temps glaciaires; tout ceci étant rendu possible depuis l'établissement de corrélations entre divers indicateurs.

Ces corrélations situent dans le temps relatif des événements identifiés en différents points du globe sur la base de caractères différents, l'ensemble formant un cadre qui a tendance à être considéré comme non seulement immuable mais aussi universel. Il peut alors facilement être omis que ce cadre regroupe des informations hétéroclites, établies de façon indépendante, et que certaines d'entre elles peuvent n'avoir que valeur locale (Blaauw 2012).

\section{2 | ZONATION POLLINIQUE ET CHRONOZONATION DE LA FIN DU DERNIER GLACIAIRE}

Question chronologie et environnement du Tardiglaciaire, il est souvent fait référence à la zonation pollinique établie en 1954 par Iversen à partir de données de sites danois (voir aussi, au sujet notamment des règles à suivre pour son établissement, Mangerud et al. 1974). La présence non permanente d'une plante qui se développe aujourd'hui dans des contrées de haute latitude et dans les montagnes, Dryas octopetala : le Dryas à huit pétales, a conduit à retenir son nom de genre pour qualifier les trois derniers événements froids de la fin des temps glaciaires, ceux-ci étant séparés par des périodes plus douces, Bølling et Aller $\varnothing d$, noms de localités où devraient avoir été mises en évidence des séquences polliniques caractéristiques. Presque immédiatement cependant, ces termes, qui au départ qualifiaient des biozones ou horizons (des tranches de terrain) livrant chacune une association pollinique particulière, prirent une signification climatique : Dryas I = période froide $; \boldsymbol{B} \phi$ lling = période au climat adouci ; Dryas $\|$ = période froide, ..; il fut alors admis que chaque entité pouvait être identifiée sur un espace beaucoup plus large que celui sur lequel la biozonation d'origine était fondée : le Danemark. En 2000, la succession Dryas ancien (ou Dryas I)- B $\phi$ lling- Dryas moyen (ou Dryas II)- Allerød- Dryas récent (ou Dryas III) est reconnue sur une aire géographique dépassant largement les limites du Danemark, s'étendant vers le sud, au moins jusqu'au niveau de la chaîne jurassienne ( Richard et al. 2000). Cependant, le Dryas moyen étant difficile à percevoir, aujourd'hui on retient généralement pour le Tardiglaciaire la succession: Dryas ancienBølling/Aller $\phi \mathrm{d}$ - Dryas récent, succession qui serait identifiable au moins à l'échelle européenne, chaque entité ayant conservé sa signification climatique.

Sous l'influence de facteurs climatiques, la végétation s'étage suivant tant l'altitude que la latitude : il fait toujours plus froid aux pôles qu'à l'équateur, plus froid aussi au sommet des montagnes qu'au bord de l'océan. En conséquence, les limites chronologiques d'une entité climatique définie par des caractères biologiques ne peuvent être synchrones à l'échelle d'un continent voire d'une région au relief accentué. Ces vraisemblables décalages chronologiques n'ont jamais été niés. Cependant le fait de paralléliser la succession Dryas
ancien-B $\phi$ lling/Aller $\varnothing \mathrm{d}$-Dryas récent avec des indicateurs couvrant de plus longues périodes conduit à situer précisément les limites (début et fin) de chaque épisode : le Dryas ancien se développerait entre -18000 et 15600 ans (comme le Stadiaire Groenlandais 2a (GS 2a); après une période de transition qui durerait près de 1000 ans, le B $\phi$ lling/Aller $\varnothing d$ débuterait à -14640 ans et se poursuivrait jusqu'à -12740 ans avant l'Actuel (comme l'Interstadiaire Groenlandais 1 (ou GI 1). Suivrait le Dryas récent, dernière phase du Tardiglaciaire, qui perdurerait un peu plus de un millénaire ainsi que le Stadiaire Groenlandais 1 (ou GS 1) (voir notamment Rasmussen et al. 2006).

La succession Dryas ancien-Bфlling/Allerød-Dryas récent, devient ainsi une succession de chronozones, chacune étant caractérisée par sa situation dans le temps (son âge) et non plus par sa végétation.

Du fait de la multiplication des datations, les ensembles archéologiques (ou l'élément lui-même qui a été daté) peuvent être replacés dans cette succession sur la seule force des dates. Ceci fait, si l'on oublie que l'on se réfère à une chronozonation, on admet, à tort, que chaque ensemble daté s'est formé sous les conditions environnementales qui ont régi la formation de la biozone du même nom. Par exemple :

1- Dans un travail discutant de nouvelles dates obtenues pour des ossements de Harfang, Renne et Cerf provenant de sites magdaléniens du Sud-ouest de la France (Szmidt et al. 2009), on trouve l'assertion suivante : «.. les dates du Renne obtenues à Duruthy (c. 3) et Dufaure (c. 3) se rattachent au $B \varnothing l$ lling et fragilisent fortement l'hypothèse d'une perduration de l'espèce à l'Allerød au sud du Sable des Landes ( Costamagno et al. sous presse).» (Szmidt et al. 2009 - p. 583 - l'article Costamagno et al. cité est paru en 2016-). Si l'on raisonne en chronostratigraphe, le deuxième membre de phrase de cette affirmation est presque trop doux; "fragilisent fortement l'hypothèse ..» pourrait être remplacé par "permettent de rejeter l'hypothèse....»: les dates se situant dans l'intervalletemps qui est accordé au Bølling et non à l'Aller $\phi d$. Cependant, si l'on raisonne en biostratigraphe ce même membre de phrase n'a pas lieu d'être : une date n'a pas vocation à critiquer une hypothèse fondée sur des principes écologiques. On peut écrire que l'on n'a pas (encore ?) de preuve de la présence du Renne dans les Pyrénées en deçà de moins 14000 ans mais cela ne peut remettre en cause l'hypothèse selon laquelle, lors des radoucissements climatiques, les aires de répartition des végétaux, comme celles des ongulés qui leur sont liées, s'étalent à la fois vers les hauteurs et vers le nord jusqu'à se disjoindre (voir Ramade, 1984 - p. 42 pour la distribution actuelle des biocœnoses ; p. 295 pour les analogies entre leurs successions dans le temps et celles observées en fonction de leur localisation géographique). Si l'on rencontre aujourd'hui encore dans nos montagnes des végétaux et des animaux qui sont également présents dans le Grand Nord, c'est que leurs aires de répartition s'est scindée après avoir occupé les zones basses de nos régions (cas du «fameux » Dryas à 8 pétales, du Pic Noir, du Lagopède, du Lièvre variable, de l'Hermine...). Cependant, les zones habitables étant beaucoup moins étendues en montagne qu'en plaine, tous (notamment les plus gros demandeurs d'espaces) ne peuvent y survivre 
longtemps. Le Renne, notamment, peu adapté à cet environnement, a sans doute rapidement laissé place aux animaux naturellement montagnards et rupicoles tels le Chamois et le Bouquetin.

2 - Dans le même article, Szmidt et al. (2009) écrivent page 585 : "Pour Le Morin, ... le niveau BI date d'environ 13000 BP tandis qu'A IV remonte à 12700-12400 BP. Mais la calibration conduit à un recouvrement partiel des dates... La transition d'un milieu ouvert et froid dominé par le Renne à un milieu un peu moins froid mais surtout plus humide dans lequel le Cerf se redéploye,.... se place donc vers 15200-15000 cal. BP. Cet intervalle se situe à l'extrême limite inférieure du début du Bølling, $14692 \pm 186$ b2k (années avant 2000 AD), telle que définie par la chronologie glaciaire GICCO5 ... ». Là, il n'est bien question que de datations; elles ont permis de replacer dans le temps, à très peu près, à quel moment les conditions climatiques ont changé dans une zone géographique donnée : les collines aquitaines.

Au siècle passé, avant la multiplication des datations, quand on utilisait les termes Dryas ancien, B $\varnothing$ lling, Dryas moyen, Allerød ou Dryas récent, on parlait de climatozone (ou biozone caractérisée par une association faunique ou floristique à signification climatique). Aussi, le fait d'ajouter que ce changement environnemental se place au tout début du Bфlling (entité chronologique) peut conduire les «anciens auteurs» à retenir que le passage du «froid au moins froid» - c'est-à-dire l'arrivée du B $\phi$ lling, entité climatique - se situe, dans l'espace européen, vers $14692 \pm 186$ b2k (années avant 2000 AD). Je ne suis pas sûre que c'était le cas dans les pays nordiques au même moment.

Il est vrai que, lorsque les questions qui se posent en Archéologie demandent des réponses à faible résolution chronologique, ce niveau d'imprécision peut être ignoré. Cependant, il n'en va pas de même quand des réponses plus précises sont attendues comme celles propres à l'environnement des «derniers chasseurs de Renne» de l'Aquitaine. L'indistinction entre climatozone et chronozone pose aussi problème lorsqu'il s'agit d'utiliser les données des travaux anciens qui ne connaissaient pas les unités chronologiques. Peut-être serait-il préférable de n'utiliser ces termes que dans leur sens premier.

\section{3 | LE GISEMENT DE PONT-D'AMBON, COMMUNE DE BOURDEILLES, DORDOGNE}

Dès sa découverte, le gisement de Pont-d'Ambon fut reconnu comme formé postérieurement aux gisements magdaléniens «classiques» du Périgord : ceux-ci livraient une faune froide, à Renne dominant le cortège des ongulés, celui-là livrait une faune tempérée à Cerf dominant (couche 4, 3b, 3a et 3); la couche 2 se démarquait cependant quelque peu (Delpech 1983).

Les travaux de fouilles menés à la suite montrèrent l'hétérogénéité de la couche 4 dont seule la base pouvait être à très peu près contemporaine des niveaux magdaléniens les plus récents: la base de la couche 4 (4 inf.) livrait, en effet, des Rongeurs «froids» (Marquet 1989) et, en 1984, un bois de Renne débité en était exhumé (fig.1). Les couches $3 b, 3 a$ et 3 contenaient les restes d'une faune de forêt tempérée dont on n'avait peu (ou pas ?) d'exemples dans les gisements de la région; elle livrait, en outre, une quantité élevée de restes de Lapin (Jones 2009) et de poissons (Le Gall 1984) indiquant que les occupants avaient fondé leur diète plus sur ces taxons que sur les ongulés. La couche 2 différait quelque peu des couches sous-jacentes : Lapin et poissons étaient moins nombreux et les ongulés de forêt (Cerf, Chevreuil et Sanglier) laissaient place aux ongulés de milieu ouvert (Cheval et Aurochs ), suite d'un phénomène amorcé dès la formation de la couche 3. Le Cerf de la couche 2 était un grand Cerf (Gilbert 1984). Or, dans une faune du Pléistocène supérieur, la présence d'un grand (ou très grand) Cerf est souvent révélatrice de conditions froides: durant le Pléistocène supérieur, le Cerf n'a jamais quitté les plaines et coteaux d'Aquitaine mais, au cours des périodes les plus rigoureuses, les quelques groupes qui y ont survécu sont presque toujours composés de sujets de taille grande à très grande (Delpech et Prat 1980). Autre ongulé représenté dans la couche 2 : le Chamois. Il y a laissé un fragment proximal de métacarpe droit débité (référencé $\mathrm{H} 8 \mathrm{n}^{\circ} 33$ ) et, sans doute, un sésamoïde référencé " $K 9$ couche 2 remaniée » (détermination qui pourrait être à revoir car effectuée à partir d'un dessin du Directeur des fouilles, Guy Célérier) (fig.2). Il y a toutefois de bonnes chances que ce sésamoïde se rapporte au même animal (et à la même patte) que le fragment proximal de métacarpe : il porte en effet de nombreuses et profondes stries qui pourraient avoir été faites lors du travail effectué pour détacher de longues longuettes osseuses sur le métacarpe ; alors qu'il était encore en position anatomique, ce sésamoïde aurait reçu l'aboutissement des efforts de rainurage. Aussi, nous admettons ici que cette patte provient d'un animal tué localement et retenons l'hypothèse selon laquelle, lors de la formation de la couche 2, l'aire de répartition du Chamois, forme de montagne, s'étendait sur une zone d'altitude relativement basse. À l'évidence, les ongulés de la couche 2 de Pont-d'Ambon forment une association de milieu ouvert froid mais, dans le cortège, il manque l'espèce phare: le Renne, et celle des milieux ouverts steppiques : le Bison. Pour l'un comme pour l'autre, l'aire de répartition ne s'étale plus jusqu'aux confins sud-ouest de la Grande Plaine nord-européenne ; celle du Bison est recentrée sur les plaines steppiques nord-européennes et asiatiques et celle du Renne sur des régions plus nordiques encore; l'un et l'autre n'ont pu (ou n'ont pas eu le temps d') étendre de nouveau leurs aires d'habitat jusqu'en Périgord. Autre particularité de la couche 2, la présence de restes de Chien (Célérier et Delpech 1978 ; Boudadi-Maligne et al. 2018).

Ces points ont été détaillés dans l'un des articles (Delpech 2018) qui composent l'ouvrage paru en hommage à Guy Célérier (Averbouh et al. 2018) dans lequel j'avais conclu à un âge Alleröd pour les couches 4 (sans 4 base), 3b, 3a et 3 , et un âge Dryas récent pour la couche 2. Une illustration y présentait la position chronologique d'événements-clés et ses implications environnementales relativement à la courbe NGRIP et à la chronozonation de la fin des temps glaciaires; ces propositions reposaient sur les datations connues du gisement de Pont-d'Ambon et de quelques sites du Sud-ouest de la France (Delpech op. cit.- fig. 1). 

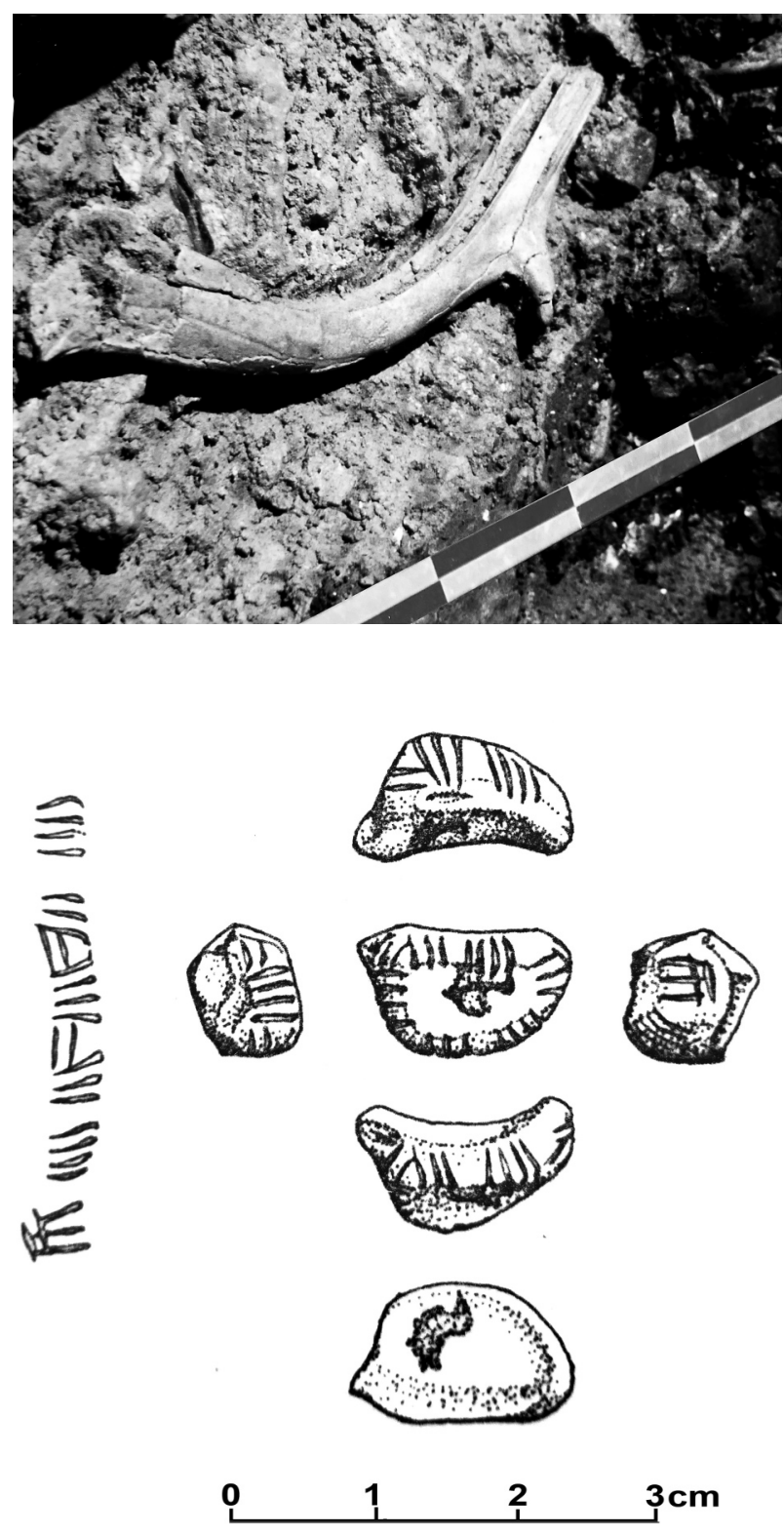

\section{4 | BIOSTRATIGRAPHIE DE LA FIN DES TEMPS GLACIAIRES POUR UN GRAND SUD-OUEST}

Ici, c'est à partir des relations biostratigraphiques suggérées par l'association des ongulés et par quelques taxons caractéristiques que seront établies les relations chronologiques. Les datations seront considérées dans un second temps. Ont été pris en compte les mêmes gisements que dans l'article sus cité à savoir, outre le Pont-d'Ambon, les gisements de Bois Ragot (Gouex, Vienne) (Gilbert 1984; Griggo 1996), Duruthy (Sorde-l'Abbaye, Landes) (Delpech 1967, 1983), Faustin (Cessac, Gironde) (Delpech 1971), La Gare de Couze (Lalinde, Dordogne) (Delpech 1967, 1983), Grotte XVI (Cénac-et-Saint-Julien, Dordogne) (Grayson et Delpech 2003), La Madeleine (Tursac, Dordogne) (Delpech 1983), Le Morin (Pessac-sur-Dordogne; Gironde) (Delpech 1967, 1983) (cf. carte dans la fig.3). Ce sont des gisements qui, tous, ont fait l'objet de révision et d'études nouvelles, notamment et pour beaucoup, dans le cadre du projet Magdatis « Le Magdalénien de la façade atlantique face aux changements environnementaux» (voir Laroulandie et al. 2017 pour un « retour » sur ce projet).

\section{FIGURE 1}

Bois de Renne débité découvert en 1984 dans le carré J7, couche $4 \mathrm{i}$ (4 inf.), du gisement de Pont d'Ambon (Bourdeilles, Dordogne). Photo Guy Célérier.

Cut reindeer antler discovered in 1984 in Pont d'Ambon square J7, bed $4 \mathrm{i}$ (4 inf.).

\section{FIGURE 2}

Sésamoïde de Chamois découvert dans le gisement de Pont d'Ambon, carré K, couche 2 (remaniée ?). Dessin Guy Célérier.

Chamois sesamoid found at Pont d'Ambon, square $K$, bed 2 (reworked). Illustration by Guy Célérier.

L'association des ongulés et les formes caractéristiques présentes dans une couche (ou un niveau, ou un ensemble de couches selon les données disponibles) constituent les bases de réflexion de ce travail (tabl. 1). C'est à partir du nombre de restes déterminés de chaque ongulé qu'a été bâtie la figure 3. Chaque ensemble stratigraphique y est représenté par un anneau formé de plusieurs éléments colorés; chaque élément est propre à un ongulé et son développement proportionnel au nombre de ses restes relativement au nombre total de restes d'ongulés déterminés ; au centre de chaque anneau sont indiqués la couche et le nombre total de restes d'ongulés déterminés. Pour ces données ont été consultés les travaux cités plus haut ainsi que, pour les gisements de Bois-Ragot, Faustin et Le Morin, respectivement, Chevallier 2015 ; Feyfant et al. 2015 et Mallye et al. 2018. L'importance de chaque groupe écologique a été considérée en priorité : groupe de milieu ouvert arctique (GMOA) avec le Renne comme chef de file, groupe de milieu ouvert non arctique (GMONA) avec Bison, Bœuf (ou Aurochs) et Cheval et groupe de milieu boisé (GMB) avec Cerf, Chevreuil et Sanglier (pour plus de précisions voir notamment Guadelli 2011). Sur la fig. 3, la 


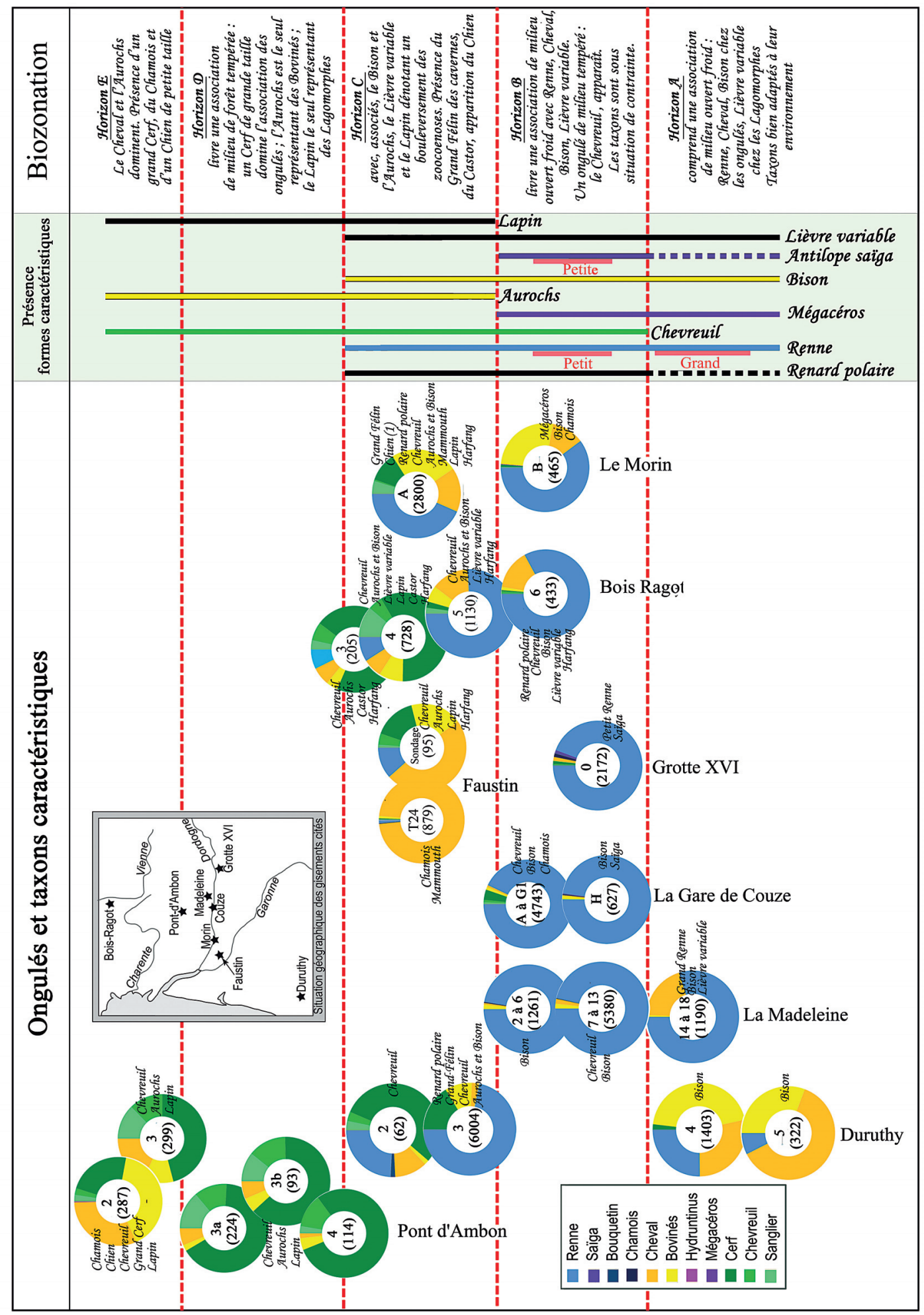

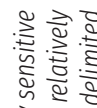

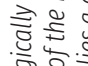

so

웜

ธั ॐ

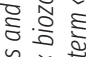

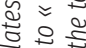

항

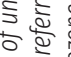

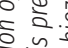

는

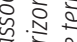

ปे

\#

¿ू हू

过

实

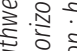

일

竞吉

웜. ㅎำ

혼

원

¿

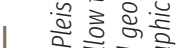

m 苟产

山

愛 佂 这旅

동

뜽 응

鱼造

등크

ฯ

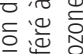

完产

岕芯药

ن่

뜐 츰

8

苍密

위의

जे

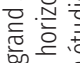

亏. 등

苍

产这

훈 음 흥

品鱼产

合产

语

ป ญ 巳

들

ब

으 흏흔

政

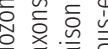




\begin{tabular}{|c|c|c|c|c|c|c|c|c|c|c|c|c|c|}
\hline & \multicolumn{4}{|c|}{$\begin{array}{c}\text { Bois Ragot } \\
\text { (Gilbert 1984, Griggo 1996) }\end{array}$} & \multicolumn{5}{|c|}{$\begin{array}{c}\text { Duruthy } \\
\text { (Delpech } 1975,1983 \text { ) }\end{array}$} & \multicolumn{2}{|c|}{$\begin{array}{c}\text { Faustin } \\
\text { (Delpech 1971, Feyfant et al. 2015) }\end{array}$} & \multicolumn{2}{|c|}{$\begin{array}{c}\text { Gare de Couze } \\
\text { (Delpech 1975, 1983) }\end{array}$} \\
\hline & 3 & 4 & 5 & 6 & 2 & 3 & $3^{\prime} *$ & 4 & 5 & Sondage & travée 24 & A à G1 & H \\
\hline Sanglier & $\begin{array}{c}7 \\
(3,41)\end{array}$ & $\begin{array}{c}82 \\
(11,25)\end{array}$ & $\begin{array}{c}23 \\
(2,03)\end{array}$ & 0 & $\begin{array}{c}2 \\
(3,23)\end{array}$ & $\begin{array}{c}1 \\
(0,02)\end{array}$ & & & & $\begin{array}{c}1 \\
(1,06)\end{array}$ & & $\begin{array}{c}31 \\
(0,66)\end{array}$ & $\begin{array}{c}1 \\
(0,16)\end{array}$ \\
\hline Chevreuil & $\begin{array}{c}14 \\
(6,83)\end{array}$ & $\begin{array}{c}39 \\
(5,35)\end{array}$ & $\begin{array}{c}2 \\
(0,18)\end{array}$ & $\begin{array}{c}4 \\
(0,92)\end{array}$ & $\begin{array}{c}2 \\
(3,23)\end{array}$ & $\begin{array}{c}2 \\
(0,03)\end{array}$ & & & & $\begin{array}{c}4 \\
(4,21)\end{array}$ & & $\begin{array}{c}41 \\
(0,87)\end{array}$ & \\
\hline Cerf & $\begin{array}{c}146 \\
(71,22)\end{array}$ & $\begin{array}{c}427 \\
(58,57)\end{array}$ & $\begin{array}{c}25 \\
(2,21)\end{array}$ & $\begin{array}{c}2 \\
(0,46)\end{array}$ & $\begin{array}{c}34 \\
(54,84)\end{array}$ & $\begin{array}{c}972 \\
(16,19)\end{array}$ & & $\begin{array}{c}26 \\
(1,85)\end{array}$ & & $\begin{array}{c}15 \\
(15,79)\end{array}$ & $\begin{array}{c}5 \\
(0,57)\end{array}$ & $\begin{array}{c}179 \\
(3,77)\end{array}$ & \\
\hline Mégacéros & & 0 & & & & & & & & & & & \\
\hline Hydruntinus & & $\begin{array}{c}1 \\
(0,14)\end{array}$ & & & & & & & & & & & \\
\hline Bœuf ou(et) Bison & $\begin{array}{c}9 \\
(4,39)\end{array}$ & $\begin{array}{c}64 \\
(8,78)\end{array}$ & $\begin{array}{c}67 \\
(5,93)\end{array}$ & $\begin{array}{c}5 \\
(1,15)\end{array}$ & $\begin{array}{c}1 \\
(1,61)\end{array}$ & $\begin{array}{c}564 \\
(9,39)\end{array}$ & $\begin{array}{l}3 \\
--\end{array}$ & $\begin{array}{c}628 \\
(44,76)\end{array}$ & $\begin{array}{c}99 \\
(30,75)\end{array}$ & $\begin{array}{c}18 \\
(18,94)\end{array}$ & $\begin{array}{c}8 \\
(0,91)\end{array}$ & $\begin{array}{c}49 \\
(1,03)\end{array}$ & $\begin{array}{c}7 \\
(1,12)\end{array}$ \\
\hline Cheval & $\begin{array}{c}14 \\
(6,83)\end{array}$ & $\begin{array}{c}50 \\
(6,86)\end{array}$ & $\begin{array}{c}154 \\
(13,63)\end{array}$ & $\begin{array}{c}63 \\
(14,56)\end{array}$ & $\begin{array}{c}7 \\
(11,29)\end{array}$ & $\begin{array}{c}143 \\
(2,38)\end{array}$ & $\begin{array}{l}32 \\
--\end{array}$ & $\begin{array}{c}396 \\
(28,23)\end{array}$ & $\begin{array}{c}199 \\
(61,8)\end{array}$ & $\begin{array}{c}46 \\
(48,42)\end{array}$ & $\begin{array}{c}853 \\
(97,04)\end{array}$ & $\begin{array}{c}32 \\
(0,67)\end{array}$ & $\begin{array}{c}2 \\
(0,32)\end{array}$ \\
\hline Chamois & & 0 & $\begin{array}{c}1 \\
(0,09)\end{array}$ & & & $\begin{array}{c}3 \\
(0,05)\end{array}$ & & & & & $\begin{array}{c}2 \\
(0,23)\end{array}$ & $\begin{array}{c}1 \\
(0,02)\end{array}$ & $\begin{array}{c}2 \\
(0,32)\end{array}$ \\
\hline Bouquetin & & 0 & & & $\begin{array}{c}1 \\
(1,61)\end{array}$ & $\begin{array}{c}13 \\
(0,22)\end{array}$ & & & & & & & $\begin{array}{c}1 \\
(0,16)\end{array}$ \\
\hline Saïga & & 0 & & & & & & & & & & & $\begin{array}{c}1 \\
(0,16)\end{array}$ \\
\hline Renne & $\begin{array}{c}15 \\
(7,32)\end{array}$ & $\begin{array}{c}66 \\
(9,05)\end{array}$ & 75,93 & $\begin{array}{c}359 \\
(82,91)\end{array}$ & $\begin{array}{c}15 \\
(24,19)\end{array}$ & $\begin{array}{c}4306 \\
(71,72)\end{array}$ & $\begin{array}{l}11 \\
--\end{array}$ & $\begin{array}{c}353 \\
(25,16)\end{array}$ & $\begin{array}{c}24 \\
(7,45)\end{array}$ & $\begin{array}{c}11 \\
(11,58)\end{array}$ & $\begin{array}{c}10 \\
(1,14)\end{array}$ & $\begin{array}{c}4410 \\
(92,98)\end{array}$ & $\begin{array}{c}613 \\
(97,76)\end{array}$ \\
\hline Mammouth & & & & & & & & & & & $\begin{array}{c}1 \\
(0,11)\end{array}$ & & \\
\hline $\begin{array}{l}\text { Nombre total de } \\
\text { restes d'ongulés } \\
\text { déterminés }\end{array}$ & 205 & 729 & 1130 & 433 & 62 & 6004 & 46 & 1403 & 322 & 95 & 879 & 4743 & 627 \\
\hline
\end{tabular}

* ensemble non figuré (trop peu de restes déterminés)

\section{- TABLEAU 1 -}

Les ongulés de huit gisements du Grand Sud-ouest ; nombre de restes identifiés et proportion par couche et par taxon.
Ungulates in eight sites in Southwestern France; Number of identified specimen and proportion by layer and by taxon. couleur bleue représente le GMOA, les tons jaune et orangé le GMONA et les tons verts le GMB ; ainsi la couleur dominante d'un anneau peut donner une information rapide sur le milieu environnant. Par aileurs, ont été considérés comme formes caractéristiques 1) des taxons aujourd'hui inféodés à un milieu précis dont l'aire de répartition s'est fortement déplacée lors des changements climatiques, par exemple un taxon des forêts tempérées, le Chevreuil, un taxon des régions semi-désertiques (l'Antilope saïga) ou encore des taxons du grand nord arctique ou (et) de haute montagne comme le Renne mais aussi le Chamois, le Lièvre variable et le Renard polaire, 2) un taxon disparu, animal symbolique des temps froids pléistocènes, le Mammouth, 3) des taxons, d'un même groupe taxonomique, dont on sait, par expérience, qu'ils n'ont pas exactement les mêmes exigences de milieu, c'est-à-dire que leurs aires de répartition pouvaient se chevaucher mais la zone de chevauchement se déplaçait lors des changements climatiques; ce sont, chez les Bovinés, le Bison et le Bœuf (Aurochs) et chez les Léporidés, le Lapin et (de nouveau) le Lièvre variable. La présence de ces «formes caractéristiques» est indiquée, en clair, contre chaque anneau. Pour chaque gisement, l'ordonnancement des couches est préservé : les anneaux les plus bas qui représentent les couches inférieures sont surmontés par ceux qui figurent les couches supérieures. Concernant le gisement de Faustin, deux séries osseuses le représentent, l'une provient d'un sondage préliminaire aux fouilles (Delpech 1971), l'autre d'un secteur délimité du gisement: la travée 24 (T24) (Feyfant et al. 2015) ; censées avoir été tirées d'un même ensemble stratigraphique, elles ont été placées au même niveau. Les ensembles Al, All, (AI-II), AllI et A IV du gisement du Morin ont été regroupés : ces subdivisions avaient été établies lors des fouilles selon la profondeur dans un ensemble homogène visuellement (ainsi que nous l'avait précisé, en son temps, le professeur F. Bordes). De même pour les subdivisions A à $\mathrm{H}$ de la Gare de Couze ; toutefois nous avons isolé la couche $\mathrm{H}$ de l'ensemble A à G1 en raison de la présence de l'Antilope saïga.

La faune du gisement du Morin, déterminée il y a près d'un demi-siècle (Delpech 1967, 1975, 1983), a fait l'objet de réévaluations qui apportent peu de changement au spectre des ongulés; s'y ajoutent cependant deux taxons: le Mammouth représenté par des lames d'ivoire et le Chamois par une cheville osseuse (Mallye et al. 2018). En outre, les derniers travaux révèlent la présence d'un Canidé à forte incidence sur le mode de vie des hommes magdaléniens : le Chien (Boudadi-Maligne et al. 2012); ses restes avaient été attribués au Loup (Delpech op.cit,) ; une remarque soulignait toutefois la petite taille de quelques vestiges dont, notamment, une carnassière inférieure (Suire 1969). Sur la figure 3, pour représenter le gisement du Morin, ce sont les chiffres établis en 1975 qui ont été utilisés; la présence des nouveaux taxons identifiés, Mammouth en $\mathrm{A}$ et Chamois en $\mathrm{B}$, y est indiquée en clair.

Les diverses couches (ou « niveaux ») des 8 gisements du grand Sud-ouest ont été distribuées dans 5 horizons (ou biozones) (fig. 3) ; chacun d'eux regroupe des couches dont la composition taxonomique révèle une proximité environnementale. Ce sont, de la base au sommet:

Horizon A: La Madeleine 14 à 18, Duruthy 4, Duruthy 5 Les formes de milieu ouvert non arctique sont bien représentées (Cheval à La Madeleine) voire dominent (Bison + Cheval à Duruthy). Le Renne domine à La Madeleine. La 
taille corporelle de ce Cervidé est relativement élevée dénotant des conditions environnementales qui lui étaient particulièrement favorables (Kuntz 2011 - p. 296). Le Lièvre variable est le seul représentant des Léporidés.

Horizon B : La Madeleine 2 à 13, la Gare de Couze H, La Gare de Couze A à G1, la Grotte XVI 0, Le Morin B, Bois Ragot 6 Le Renne, de petite taille (Grotte XVI) (Delpech 2018, note 7), domine de façon écrasante la cohorte des ongulés; l'Antilope saïga est présente (Couze H, Grotte XVI), elle est sans doute de petite taille comme le sont les antilopes saïgas du gisement du Quéroy en Charente qui se place vraisemblablement dans la même biozone (voir à ce sujet Costamagno 2001 - p. 117, note 2). Les Bovinés ne sont représentés que par le Bison. Chamois, Lièvre variable, Renard polaire et Harfang sont présents. Notons la présence du Chevreuil (La Madeleine 7 à 13, La Gare de Couze A à G1, Bois Ragot 6) ainsi que celle du Mégacéros (Le Morin B).

Horizon C: Duruthy 2, Duruthy 3, Bois Ragot 5, Bois Ragot 4, Bois Ragot 3, Le Morin A, Faustin Sondage, Pont-d'Ambon 4 base (cf.supra).

Dans cet horizon, on retrouve l'association de l'horizon sous-jacent, Antilope saïga et Mégacéros exceptés, avec des formes retirées aujourd'hui dans les régions steppiques froides qui peuvent pulluler comme le Lièvre variable (Bois Ragot 5) ou le Harfang des neiges (Bois Ragot 5, Le Morin A); plus rares sans doute mais encore représentés, le Mammouth ainsi que le Chamois (Faustin T24). Les formes de forêt, Cerf, Chevreuil sont bien présentes et le Bœuf primitif (ou Aurochs) apparaît (Duruthy 3, Faustin Sondage, Bois Ragot 5, Bois Ragot 4, Le Morin A). Le Lapin, nouveau venu (Faustin sondage, Bois Ragot 5, Le Morin A), se retrouve aux côtés du Lièvre variable (Bois Ragot 5, Bois Ragot 4). Signalons aussi la présence au Morin et à Duruthy (Morin A, Duruthy 3 ) de ce qui est sans doute l'un des derniers représentants européens de la lignée du Grand Félin des cavernes (Delpech 1975 - p. 133 ; 1983 pl. 6 ; Fosse et al. 2017) ainsi que celle du Chien (Morin A) (Boudadi-Maligne et al. 2012).

Horizon D : Pont-d'Ambon 4, Pont d'Ambon 3b, Pont d'Ambon 3a.

Cet horizon livre une faune de forêt tempérée (Cerf, Chevreuil et Sanglier) bien différente de celles des niveaux sous-jacents. Cheval et Aurochs sont représentés, celui-ci devenant le seul représentant des Bovinés. Le Lièvre variable est absent et les restes de Lapin abondent.

Horizon E: Pont-d'Ambon 3, Pont d'Ambon 2.

La faune de forêt tempérée cède la place aux formes de milieu ouvert non arctique : Cheval et Aurochs, progressent fortement et l'Aurochs, pour la première fois (à notre connaissance dans un site avec Paléolithique supérieur) domine l'association des ongulés (Pont d'Ambon 2). Présence du Chamois et du Chien (Pont d'Ambon 2). Les lapins sont moins nombreux.

Pendant la période de formation de ces cinq horizons, la présence des taxons retenus comme " formes caractéristiques » ne se distribue pas au hasard (fig.3). L'Antilope saïga disparaît lors de la formation de l'Horizon B; le Renne, le Renard polaire et le Lièvre variable lors de la formation de l'Horizon C. D'autres taxons apparaissent: le Chevreuil avec l'Horizon B, l'Aurochs et le Lapin avec l'Horizon C.

Alors que dans l'Horizon A sont représentés des taxons formant une association pléistocène «classique», bien adaptée à son environnement (taille corporelle du Renne assez élevée), dans l'Horizon B, il n'en va plus de même ; des taxons apparaissent (Chevreuil), d'autres souffrent dans un environnement qui ne leur convient plus (Renne de petite taille corporelle) avant de disparaître (Antilope saïga). L'Horizon C se forme durant la période aux plus forts changements environnementaux ; c'est dans cet Horizon que se placent le relais du Bison par l'Aurochs et celui du Lièvre variable par le Lapin; le Chamois est toujours présent aux côtés du Bison, du Renne et du Renard polaire ; on y relève aussi la présence du Grand Félin des cavernes, du Mammouth et celle du Chien. Lors de la formation de l'Horizon D, la grande faune pléistocène a disparu, la forêt tempérée est en place et le Cerf le chef de file des ongulés. Avec l'Horizon E, les conditions froides se manifestent de nouveau ; la réouverture des milieux se marque par le développement du Cheval et de l'Aurochs mais le Renne ne réinvestit pas l'Aquitaine.

L'examen de la figure 3 conduit à soulever un dernier point concernant le groupe de milieu ouvert non arctique (figuré en orange et jaune). En effet, Cheval et Bovinés se font remarquer par leur abondance dans quelques gisements, à savoir le Morin et Faustin ainsi que dans les niveaux inférieurs de Duruthy et supérieur de Pont d'Ambon. Cette abondance n'est pas liée à un Horizon mais pourrait être en relation avec la topographie de la région qui, suivant le climat du moment, peut favoriser le développement de steppes nourricières dans les plaines et collines girondines ou dans les premiers contreforts montagneux des Pyrénées et du Massif central. Cependant, dans l'un des deux échantillons du gisement girondin de Faustin : Faustin T24, les restes de chevaux constituent la quasitotalité de l'échantillon; cette abondance ne peut totalement s'expliquer par un environnement local favorable au développement des troupeaux; s'y ajoute le fait que cet Equidé a fait l'objet de chasses spécialisées (Feyfant et al. 2015) ; l'échantillon Faustin sondage donne sans doute une idée plus juste de l'association des ongulés qui se trouvaient alors dans la région.

\section{5 | DATATIONS}

Datations ${ }^{14} \mathrm{C}$ conventionnelles ou par SMA, toutes ont été prises en compte. Ainsi que l'écrit Christine Oberlin, « il est injustifié de suspecter une datation parce que la teneur en radiocarbone a été mesurée avec des appareils moins performants. Seule la précision change évidemment, mais la date reste valable » (Oberlin 2007). Sur la fig.4, les dates obtenues par la méthode conventionnelle sont facilement repérables: ce sont celles qui s'étalent le plus largement dans le temps mais il existe quelques exceptions comme par exemple la date du chien de Pont d'Ambon dont l'imprécision est importante.

Les cinq horizons précédemment définis ont été situés relativement à la courbe climatique NGRIP (Rasmussen et al. 2014) et à la succession Dryas ancien- Bölling/AllerödDryas récent (fig.4). Cette figure reprend le fond d'une illus- 


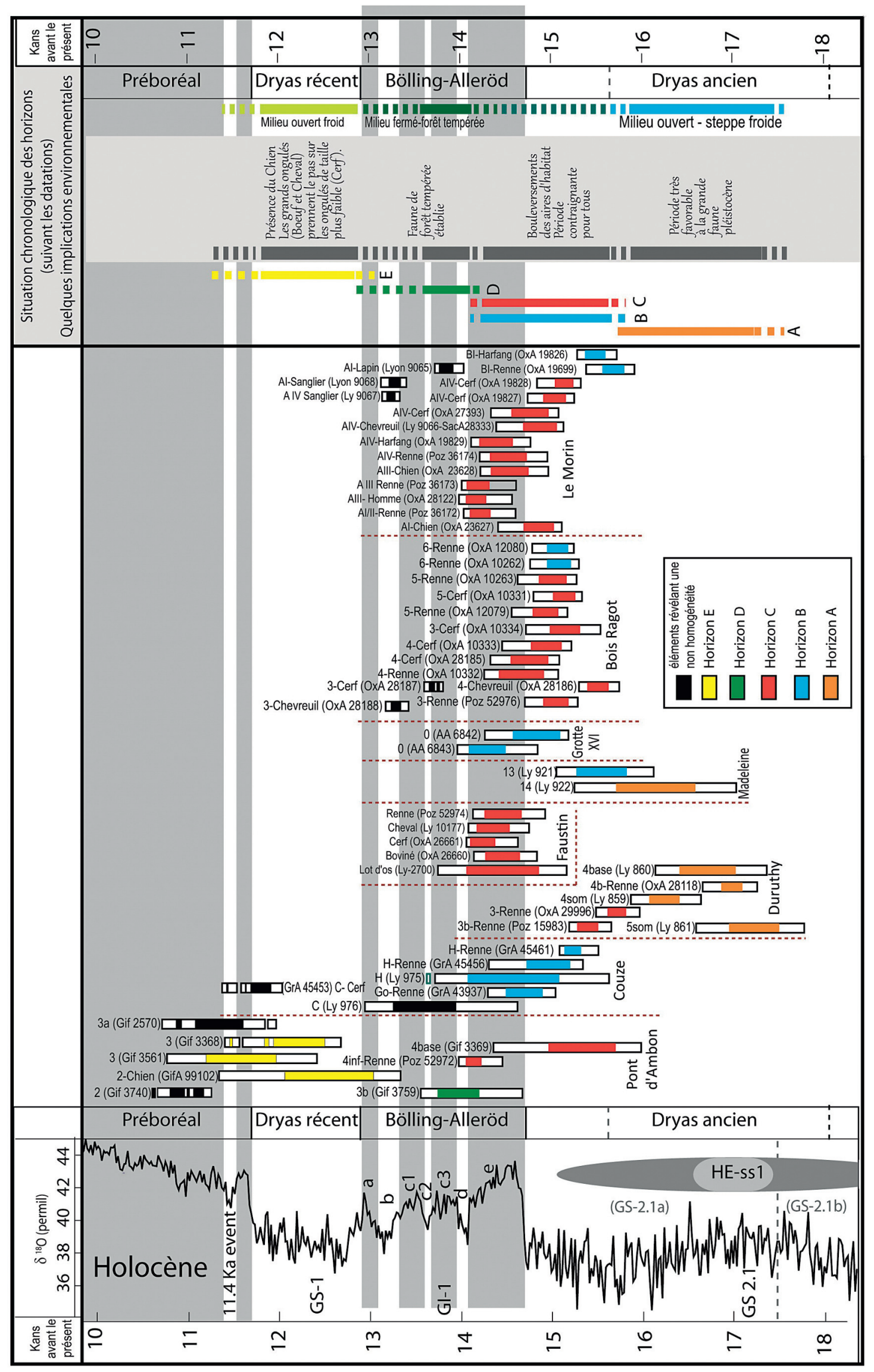


tration d'un précédent travail (Delpech 2018 fig. 1) sur laquelle ont été ajoutées les figurations d'autres datations : 8 pour le Morin (Mallye et al. 2018), 5 pour Bois Ragot (Chevallier 2015) et 3 pour Faustin (Feyfant et al. 2015); n'ont pas été représentées les dates obtenues, au Morin, pour 6 harpons et un outil biseauté (Mallye et al. op. cit.).

L'Horizon A (couches 5 et 4 de Duruthy et 14 à 18 de La Madeleine) se seraient déposées avant moins 16500 ans au cours de la période qui avait été qualifiée d'âge d'or des magdaléniens, période durant laquelle les ongulés de taille moyenne (Renne) et de grande taille (Bison, Cheval) sont en équilibre avec leur milieu (cf. Delpech et Lenoir 1996 ; Delpech 1999 ; Texier et Delpech 2011). Dans la chronozonation de la fin des temps glaciaires, cet âge d'or se place avant la fin du Dryas ancien (cf. fig. 4) ; en 1996, nous l'avions parallélisé avec le Bølling car nous entendions, sous ce terme, une période au climat adouci permettant le développement d'une riche steppe herbeuse (Delpech et Lenoir op. cit.) ; nous n'avons pas changé d'avis et insistons sur la nécessité de préciser le caractère retenu (chronologique, climatique, biologique,...) quand on utilise les termes de la zonation pollinique originelle. La période de passage de l'Horizon A à l'Horizon $B$, repérable stratigraphiquement à Duruthy et La Madeleine se situerait vers moins 15800 ans.

L'Horizon B (La Madeleine 2 à 13, la Gare de Couze H, La Gare de Couze A à G1, la Grotte XVI 0, Le Morin B, Bois Ragot 6) et l'Horizon C (Duruthy 2, Duruthy 3, Bois Ragot 5, Bois Ragot 4, Le Morin A, Faustin, Pont-d'Ambon 4 base [?]) se seraient formés au cours de la même période, entre moins 15800 ans et moins 14200 ans (environ). C'est la période des grands bouleversements environnementaux de la fin des temps glaciaires (cf. supra) qui s'étalerait sur près de 1600 ans. Le fait de ne pouvoir différencier chronologiquement ces deux horizons en cette période troublée indique sans doute que les réactions aux changements environnementaux de chaque taxon sont ni synchrones ni identiques : au même moment les biocœnoses variaient selon l'espace considéré. Dans les plaines girondines, si l'on en juge par les datations du Morin, la couche B que nous avons située dans l'Horizon $B$ s'est bien formée avant la couche A placée dans l'Horizon C, le changement de faune qui a justifié la séparation Horizon B-Horizon C se situerait vers moins 15300-15400 ans ; au Bois Ragot, au contraire, les datations n'apportent pas confirmation de la biostratigraphie : la couche 6 (Horizon B) paraît s'être formée au même moment que les couches 5,4 et 3 (Horizon $C$ ) ; les raisons de ce «chevauchement» chronologique sont sans doute en partie liées à l'important plateau radiocarbone qui se situe dans les périodes de formation de ces deux Horizons.

L'Horizon D (Pont d'Ambon 4 voir note 2], Pont d'Ambon 3b, Pont d'Ambon 3a) et l'Horizon E (Pont d'Ambon 3, Pont d'Ambon 2) se succèdent dans le gisement de Pont d'Ambon. Les datations sont cependant trop imprécises pour dater les périodes de formation de l'un et de l'autre (rappelons que les dates de la couche 4 concernent un Horizon formé avant l'Horizon D). Tout au plus pouvonsnous indiquer que la formation de l'horizon $D$ a dû débuter vers -14000 ans au cours de l'interglaciaire groenlandais Gl1 et s'étaler sur plus d'un millénaire (?). Quant à la formation de l'horizon E qui marque la mise en place d'un refroidissement, elle se place bien pendant la période dite Dryas récent, corrélée avec l'interglaciaire groenlandais GS1 dont le début «officiel» se place un peu avant moins 13000 ans.

\section{6 | APPORT DES DATATIONS À LA MISE EN ÉVIDENCE DE PERTURBATIONS ET REMANIEMENT DANS LES ENSEMBLES ÉTUDIÉS (fig.4).}

«Tout l'intérêt d'une datation repose sur la représentativité de l'échantillon analysé» (Oberlin 2007 p. 5). Les échantillons qui ont servi aux datations obtenues par la méthode conventionnelle $\mathrm{du}{ }^{14} \mathrm{C}$ engendrent souvent des questions dans la mesure où ils sont composés d'éléments dont il est difficile d'assurer, à postériori, la provenance stratigraphique. De toute évidence, les datations ainsi obtenues pour les couches 2 (Gif 3740) et $3 a$ (Gif 2570) de Pont d'Ambon et pour la couche C de La Gare de Couze (Ly 976) révèlent une non-homogénéité chronologique des ensembles d'ossements qui ont servi aux datations, c'est-à-dire des attributions stratigraphiques erronées d'une partie de l'échantillon qui a été daté.

Lors d'une datation par SMA qui concerne un seul élément, l'erreur d'attribution stratigraphique apparaît lorsque la date obtenue s'isole nettement de celles censées dater le même événement; c'est le cas à Couze couche $C$, pour un reste de Renne (GrA 45453), au Morin couche A pour trois restes : un de Lapin (Ly 9065 [A1]) et deux de Sanglier (Ly 9068 [A1] et Ly 9067 [A4]), et à Bois Ragot couche 3 pour 2 vestiges: un de Cerf la couche 3 (OxA 28187) et un de Chevreuil (OxA 28188).

\section{Pont d'Ambon}

Cela ne remet pas en cause, pour l'horizon E représenté à Pont d'Ambon, les deux datations de la couche 3 qui sont «assez proches» de celle du petit Chien de la couche 2 : la formation de l'horizon E a eu lieu au cours du $1^{\text {er }}$ Stade Glaciaire Groenlandais GS-1 (corrélé avec le Dryas récent). une seule datation, celle de la couche $3 \mathrm{~b}$, permet de situer la formation de l'horizon D dans le premier Interstade Groenlandais GI-1, corrélé avec le B $\varnothing$ lling/Aller $\varnothing$ d ; c'est la grande faune qui permet de situer chronologiquement les couches 4, 3b et $3 a$ de Pont d'Ambon en position plus récente que les ensembles placés dans l'horizon C.

\section{La Gare de Couze}

Sans connaissance des datations de la couche C (Lyon 976) et d'un os de Cerf (GrA 45453), l'association faunique de l'ensemble A à G1 n'aurait pas permis de douter de son homogénéité chronologique. Le Cerf, au contraire, par la découverte de vestiges indiquant la présence conjointe de grands et de petits individus (Delpech 1967) révèle une hétérogénéité ; nous y reviendrons ci-après ( § 8.2.1.) 


\section{Le Morin}

Les datations soulignent la distinction entre Horizon B (couche B) et Horizon C (couche A) ; elles justifient aussi le fait de réunir en un même ensemble les subdivisions Al, All, Alll et A IV qui avaient été créées arbitrairement lors de la fouille du gisement; elles signalent, en outre, l'hétérogénéité de cet ensemble A qui comporte des éléments plus récents ne lui appartenant pas; enfin, les 3 dates récentes rejetées, elles situent la formation de Morin A (Horizon C) entre moins 15300-15400 ans (cf. § 5) et moins 14000 ans.

Dans cet ensemble A, comme à La Gare de Couze, un grand Cerf et un petit Cerf sont représentés ce qui corrobore son hétérogénéité chronologique (cf. § 8.2.1.). Les travaux menés par les préhistoriens, notamment les «typologues de pointes» (Langlais 2018), ont montré, eux aussi, qu'il y a eu des mélanges entre Magdalénien, Azilien et Laborien (Mallye et al. 2018 ). Cependant, bien que cette couche A du Morin contienne des éléments remaniés, l'association faunique qui en est donnée (i.e. la liste des taxons) est représentative de celle qui hantait la région lorsque la couche s'est formée; certes les vestiges de Lapin et de Sanglier datés ne lui appartiennent pas mais il est vraisemblable que d'autres s'y rattachent: c'est, du moins une hypothèse à ne pas rejeter d'autant que ces deux taxons sont présents dans des ensembles appartenant au même horizon $C$, notamment Faustin sondage et Bois Ragot 3,4,5.

\section{Bois Ragot}

Les datations ne permettent pas d'isoler la couche 6 (Horizon B) des couches 5, 4 et 3 (Horizon C) (cf. § 5). Elles indiquent toutefois l'hétérogénéité de la couche 3 : deux dates, l'une de Cerf, l'autre de Chevreuil, trop récentes, s'isolent du lot. S'isole aussi du lot une date, la plus ancienne, qui concerne un reste de Chevreuil provenant de la couche 4 (OxA 28186). Il s'agit d'un fragment de métatarse (réf. K5 79) dont la signature isotopique apparaît "difficilement compatible avec un Chevreuil et le rapprocherait en réalité plus volontiers du Renne» (selon Drucker in Chevallier 2015 - p. 66). Parmi les ensembles pris en compte dans ce travail, l'ensemble 7 à 14 de La Madeleine a livré un reste de Chevreuil qui pourrait être aussi ancien. Aussi, ce qui, à Bois Ragot, semble être une erreur de détermination ne remet pas en cause l'hypothèse selon laquelle le Chevreuil était présent dans un grand SudOuest de la France avant la mise en place de l'interglaciaire Gl-1 (corrélé avec le B $\varnothing$ lling-Aller $\varnothing$ d).

\section{7 | APPORTS DE CES TRAVAUX POUR LA CONNAISSANCE DES GRANDS MAMMIFÈRES DE LA FIN DES TEMPS GLACIAIRES}

\subsection{Importance du facteur espace dans l'agencement des biocœnoses}

Rappelons que les datations nous forcent à réunir dans une même période s'étendant de -15800 ans à -14200 ans environ les deux horizons B et C. Deux horizons différents repérés dans une zone géographique assez peu étendue (cf. carte dans la figure 3) devraient pouvoir être situés chronologiquement l'un relativement à l'autre et les datations devraient au moins confirmer l'ordre stratigraphique des deux gisements stratifiés qui ont aidé à les identifier : l'horizon B est sous-jacent à l'horizon C au Morin et au Bois Ragot. Ce serait oublier l'influence du facteur espace sur les zoocœnoses - d'ongulés notamment - et le fait que, même sur un petit territoire, les variations spatiales peuvent masquer les variations temporelles. Dans notre zone d'étude, pourtant peu étendue, les formes de milieu ouvert non arctique (Cheval et grands Bovidés) sont mieux représentées en bordure des Pyrénées (Duruthy), dans le Nord de la Grande Aquitaine (Bois Ragot) et dans les zones basses girondines (Le Morin, Faustin), qu'en Périgord où le Renne domine (fig. 3) (cf. § 6). Par ailleurs, du point de vue des variations temporelles, la mise en place de la forêt caducifoliée aux dépens des espaces ouverts de steppe herbeuse ne s'est pas produite instantanément et les effets contraignants de ce processus sur les ongulés, prédateurs de la strate herbacée, ont été perçus par chacun d'eux ni de la même façon ni exactement au même moment. Chacun a réagi en fonction de ses facultés d'adaptation et de ses limites de tolérance au nouvel environnement qui se met en place (Faunmap working group 1996). Travailler sur un territoire plus étroit permettrait sans doute de percevoir plus précisément ces phénomènes mais nos travaux d'aujourd'hui concernent un Grand Sud-ouest; à cette échelle, le fait que les horizons B et C n'ont pu être distingués révèlent une réaction non simultanée des ongulés aux changements environnementaux et, en ces périodes de changements voire de bouleversements environnementaux, une diversité spatiale et temporelle des zoocœnoses. Selon les datations, il semble cependant que les relations ongulés-milieu aient atteint un nouvel équilibre à peu près au même moment, (i.e. à la fin de la formation des horizons B et C) vers moins 14200 ans.

\section{2 | Variations de taille et interprétations taxonomiques}

Entre -15800 ans et -14200 ans, Isatis, Renne, Mégacéros, Bison, Antilope saïga, Lièvre variable disparaissent de la région; à l'inverse, Chevreuil, Aurochs, Lapin, viennent occuper l'espace. Les tentatives d'adaptation d'un taxon à des conditions environnementales contraignantes passent souvent par la diminution des effectifs et la diminution de la taille des individus; c'est vrai pour l'Antilope saïga (cf. § 5) et pour le Renne ; en effet, la couche 0 de la Grotte XVI livre les vestiges d'un Renne à faible stature (cf. Delpech 2018 -p. 54, note 7); de même le Renne de la Gare de Couze et celui du Morin semblent ne pas avoir été très grands (Delpech 1983). Il nous paraît intéressant de revenir maintenant sur le cas du Cerf et de soulever un voile sur le cas de quelques carnivores.

\subsection{1 | Le cas du Cerf}

Dans le paragraphe 3. traitant de Pont d'Ambon, nous avons rappelé que le Cerf n'a jamais quitté les plaines et coteaux d'Aquitaine mais, au cours des périodes les plus rigoureuses, les quelques groupes qui ont survécu dans ces milieux ouverts steppiques sont presque toujours composés de sujets de taille grande à très grande (Delpech et Prat 1980). À l'inverse, dans un environnement 
forestier, le Cerf se développe en nombre mais reste d'assez petite taille. À Combe-Grenal par exemple, au cours de l'OIS 5 (« ancien » Würm I), le Cerf a atteint une taille si faible (Prat et Suire 1971) qu'il a été classé dans une espèce nouvelle : Cervus simplicidens (Guadelli 1996); pour notre part, il s'agirait d'une petite forme qui ne mérite pas le rang d'espèce (Delpech 1988). Ainsi, à des conditions environnementales relativement rigoureuses, correspond un grand Cerf représenté par peu d'individus. À l'inverse, sous des conditions plus clémentes favorisant le développement de la forêt, le Cerf serait de petite taille mais représenté par un fort effectif. Pour les formes inféodées au froid comme le Renne, la mise en place de conditions douces entraînent une diminution de taille et d'effectif, pour une forme inféodée à la forêt, la mise en place de conditions froides (steppiques) entraîne une augmentation de taille (loi de Bergman) et une diminution d'effectifs. Quoi qu'il en soit, le fait de trouver deux formes de Cerf dans un même ensemble stratigraphique entraîne toujours une forte suspicion de perturbations stratigraphiques. C'est le cas dans les gisements de La Gare de Couze et du Morin où le Cerf est représenté par un Cerf de taille «normale» (sous-entendant un milieu boisé) parmi lesquels quelques vestiges se rapportent sans conteste à de très grands individus (sous-entendant un milieu steppique froid).

\subsubsection{Le cas des carnivores, du Loup notamment}

La diminution de la taille des individus ne touche pas que les ongulés. Un travail sur l'Hyène des cavernes conclut aussi à une diminution de la taille de ces grands carnivores en période interglaciaire (Klein et Scott 1989). C'est aussi vrai pour un autre grand carnivore, à savoir le Grand Félin des cavernes qui est représenté dans la couche 3 de Duruthy ainsi que dans la couche A du Morin (Delpech 1983). Plus petit que le Lion actuel, il pourrait s'agir de Felis spelaea var cloueti, sous-espèce créée par Boule (1892) à partir d'une mâchoire de Félin provenant d'un niveau magdalénien de la caverne du Gros Roc (commune de Douhet, Charente maritime) (Clouet 1891). Cette importante diminution de taille est sûrement en lien avec la raréfaction du gibier. En effet, la mise en place de la forêt, qu'il s'agisse de la taïga ou de la forêt caducifoliée, entraîne toujours une baisse de la biomasse des ongulés (Ramade 1984 - p. 282, Delpech 1999). Ceci nous conduit à faire part de nos interrogations concernant le Chien du gisement du Morin (Boudadi-Maligne et al. 2012). Sa détermination repose, uniquement nous semble-t-il, sur la faible dimension de ses ossements (voir à ce sujet Janssens et al. 2019). Les mêmes causes n'auraient-elles pas eu chez les Canidés les mêmes effets que chez les Félidés? Peut-on éliminer l'hypothèse selon laquelle le Canidé du Morin serait un Loup en situation de contrainte environnementale? Nous répondons par la négative.

\section{CONCLUSIONS}

Biostratigraphie et datations doivent être prises en compte conjointement lors de l'établissement des chronologies. L'une permet de faire le lien entre gisements, l'autre situe précisément dans le temps les phénomènes environnementaux propres à tous ou (et) à chacun d'eux.
C'est cette prise en compte conjointe qui nous a permis de voir ou revoir, sous un angle nouveau, quelques modalités environnementales de la fin du Pléistocène. La fin des temps glaciaires a fait l'objet de nombreux travaux impliquant de nombreux chercheurs; voir notamment les travaux présentés à Bordeaux lors de la séance de la Société préhistorique française en mai 2012 (Langlais et al. 2014). Face à ces travaux, cet article apparaît bien modeste. Cependant, pour des questions touchant la chronologie et l'environnement que formaient les grands mammifères, il a soulevé quelques points importants que je résumerai en conclusion.

1) Une fois placée dans la chronologie, la succession Dryas ancien-Bфlling/Aller $\phi$ d-Dryas récent devient une chronozonation; alors chaque terme perd la signification environnementale qu'il avait dans la zonation pollinique originelle. À défaut de le préciser, ces termes ne devraient être employés que dans leur sens premier.

2) La fin des temps glaciaires est une période de bouleversements des biocœenoses dont on cherche à établir les modalités. Celles-ci ne peuvent cependant être perçues qu'en tenant compte et du facteur temps et du facteur espace. Pour les questions temporelles, « il existe une remarquable analogie entre la succession des biocœnoses dans le temps.... et celle observée en fonction de leur localisation géographique » (Ramade 1984 - p. 295). Pour les questions spatiales, l'agencement actuel des aires de distribution peut servir, en partie, de modèle d'organisation, soulignant l'importance qu'il faut accorder à la position géographique du (des) gisement(s) étudié(s).

3) C'est l'espèce et non l'association d'espèces qui répond aux changements environnementaux (Faunal working group 1996) : il apparaît, en effet, que les périodes de migration, conséquences des déplacements d'aires de répartition, les augmentations ou baisses des effectifs, les élévations ou baisses de la stature se produisent lors de périodes différentes selon l'espèce concernée (Delpech 1999, Texier et Delpech 2011, fig.6). Parmi les ongulés végétivores c'est sans doute le Cerf et, dans une moindre mesure, le Cheval qui sont à même de mieux nous renseigner sur les grandes étapes des changements environnementaux. Ce sont, en effet, les deux seuls ongulés qui ont été, de tous temps, présents dans le Sudouest de la France ; ainsi, ils détiennent en eux des clés de lecture des phénomènes adaptatifs propres à la région et à chacune des étapes de ces changements.

4) Chez les ongulés, parmi les phénomènes adaptatifs, les variations de taille comme celles des effectifs sont les plus visibles. La diminution de la taille corporelle peut simplement signifier que l'on passe d'un milieu steppique froid à un milieu forestier au climat adouci (en relation avec la loi de Bergman). Quant à la diminution des effectifs, elle va toujours de pair avec la mise en place de la forêt, qu'il s'agisse de la taïga ou de la forêt caducifoliée.

5) Comme pour les ongulés, les changements de la fin des temps glaciaires génèrent donc de très fortes contraintes environnementales pour les carnivores car leur gibier est beaucoup moins abondant. Leur nombre sans doute et leur taille sûrement chutent. C'est ce que l'on constate chez le Grand Félin des cavernes représenté à Duruthy et au Morin. Ce peut aussi avoir entraîné la diminution de la 
taille d'un autre prédateur : le Loup. Aussi, concernant l'attribution au Chien de restes de canidés trouvés au Morin, à la question: "Peut-on vraiment éliminer l'hypothèse de leur attribution à un loup en position de contrainte ?», nous répondons par la négative.

6) Au sujet de l'Aurochs, Bos primigenius, j'ai montré en quoi sa présence était révélatrice d'un environnement différent de celui de Bison priscus, le Bison. Bien que cette présence soit difficile à établir à partir des seuls ossements, il existe d'assez nombreux critères de différenciation Bos-Bison. Aussi les recherches doivent être poussées, chaque fois que cela est possible, afin d'aller au-delà de la détermination «Grands Bovidés» (ou «Bovinés») qui n'apporte que peu d'informations sur les conditions environnementales. À la fin des temps glaciaires, dans un grand Sud-ouest de la France, la présence de l'Aurochs est confirmée à partir de moins 15000 ans environ.

Aux grands changements environnementaux de la fin des temps glaciaires, l'homme, comme les grands carnivores, a mis en place des stratégies d'adaptation qui doivent avoir quelques points communs avec celles des autres grands prédateurs. Il paraît évident que la disparition de la grande faune pléistocène de laquelle il tirait, jusquelà, une large part de son gibier a entraîné une baisse de la démographie locale; sur la figure 4, on a presque un vide d'informations entre moins 13000 et 13500 ans, à la fin de l'interglaciaire groenlandais Gl-1. L'intérêt que l'homme manifeste alors, au Pont-d'Ambon, pour les petits lagomorphes ainsi que pour les poissons d'eau douce s'explique, me semble-t-il, aisément. C'est une question de survie. Changer de mode vie n'est pas chose facile. Dans la région, le nombre des gisements dans lesquels ce tournant a été réalisé me semble assez faible ; il y a le Pont-d'Ambon et ...d'autres sites aziliens et laboriens; combien cependant?

\section{REMERCIEMENTS}

- au professeur D.K. Grayson qui a réalisé les traductions anglaises du résumé et des légendes de figures.

- aux deux relecteurs dont les remarques ont aidé à améliorer sensiblement cet article.

\section{RÉFÉRENCES BIBLIOGRAPHIQUES}

AVERBOUH A., BONNET-JACQUEMENT P., CLEYET-MERLE J.-J. (dirs.) 2018 - L'Aquitaine à la fin des temps glaciaires. Les sociétés de la transition du Paléolithique final au début du Mésolithique dans l'espace Nord aquitain. Paleo, numéro spécial.

BANKS W.E., BERTRAN P., DUCASSE S., KLARIC L., LANOS Ph., RENARD C., MESA M. 2019 - An application of hierarchical Bayesian modeling to better constraion the chronologies of Upper Paleolithic archaeological cultures in France between ca. 32,000-21,000 calibrated years before présent. Quaternary Science Reviews 220, p. 188-214.

BLAAUW M. 2012 - Out of tune: the dangers of aligning proxy archives. Quaternary Science Reviews 36, p. 38-49
BOUDADI-MALIGNE M., MALLYE J.-B., LANGLAIS M., BARSHAYSZMIDT C. 2012 - Des restes de chiens magdaléniens à l'abri du Morin (Gironde, France). Implications socio-économiques d'une innovation zootechnique. Paleo 23, p. 39-54.

BOUDADI-MALIGNE M., BONNET-JACQUEMENT P., LANGLAIS M., FERRIER J., MALLYE J.-B. 2018 - Les chiens de Pont d'Ambon : statut, contexte et implications sociétales. In : AVERBOUH A., BONNET-JACQUEMENT P., CLEYET-MERLE J.-J. (dirs.) - L'Aquitaine à la fin des temps glaciaires. Les sociétés de la transition du Paléolithique final au début du Mésolithique dans l'espace Nord aquitain. Paleo, numéro spécial, p. 67-76.

BOULE M. 1892 - Note sur une portion de mâchoire de Felis trouvée dans la caverne du Gros Roc (Charente maritime) par MM; Bordage et Clouet. L'Anthropologie, t. 3, p. 742.

CÉLÉRIER G. 1998 - L'abri sous roche de Pont d'Ambon à Bourdeilles (Dordogne, France). Perspective synthétique. Paleo 10, p. 233-264.

CÉLÉRIER G., DELPECH F. 1978 - Un chien dans l'Azilien de Pont d'Ambon (Dordogne). Bulletin de la Société Préhistorique Française, t. 75, p. 212-215.

CHEVALLIER A. 2015 - Chasse et traitement des mammifères durant le Magdalénien et l'Azilien dans le Sud-ouest de la France. La place particulière du Cerf, sous la direction de COSTAMAGNO S. et VALENTIN B. (dirs), soutenue à l'Université Paris I - Panthéon-Sorbonne le 7 décembre 2015.

CLOUET M. 1891 - Fouilles de la grotte du Gros Roc. Association Française pour l'Avancement des Sciences, séance du 23 septembre 1891, 20ème session, 1ère partie. Marseille, 1891, p. 268.

COSTAMAGNO S. 2001 - Exploitation de l'Antilope saïga au magdalénien en Aquitaine. Paleo 13, p. 111-128.

COSTAMAGNO S., BARSHAY-SZMIDT C., KUNTZ D., LAROULANDIE V., PÉTILLON J.-M., BOUDADI-MALIGNE M., LANGLAIS M., MALLYE J.-B., CHEVALLIER A. 2016 Reexamining the timing of reindeer disappearance in Southwestern France in the larger context of late glacial faunal turnover. Quaternary international 414, p. 34-61.

DELPECH F. 1967 - Recherches paléontologiques concernant quelques gisements du Magdalénien VI. Stations de la Gare de Couze (Dordogne), du Morin (Gironde) et de Duruthy (Landes). Thèse de 3e cycle; Faculté des Sciences de l'Université de Bordeaux, n 489, 202 p. de texte ronéot., $35 \mathrm{pl}$.

DELPECH F. 1971 - L'abri Faustin, commune de Cessac (Gironde). Bulletin de la Société Préhistorique Française, t. 68 , p. 328-332.

DELPECH F. 1975 - Les faunes du Paléolithique supérieur dans le Sud-ouest de la France. Thèse de Doctorat ès Sciences naturelles, Bordeaux, n 479, 374 p., 98 pl., 159 tabl.

DELPECH F. 1983 - Les faunes du Paléolithique supérieur dans le Sud-ouest de la France. Cahiers du Quaternaire, $n^{\circ}$ 6, éditions du CNRS, $454 \mathrm{p}$. 
DELPECH F. 1999 - Biomasse d’ongulés au Paléolithique et inférences sur la démographie. Paleo, nº 11, p. 19-42.

DELPECH F. 2012 - Biostratigraphie des niveaux solutréens de Laugerie-Haute (Les Eyzies, Dordogne, France). Paleo 23, p. 105-113.

DELPECH F. 2018 - L'environnement animal durant le Tardiglaciaire et le début de l'Holocène à travers l'étude des grands mammifères de Pont d'Ambon. In : AVERBOUH A., BONNET-JACQUEMENT P., CLEYET-MERLE J.-J. (dirs) - L'Aquitaine à la fin des temps glaciaires. Les sociétés de la transition du Paléolithique final au début du Mésolithique dans l'espace Nord aquitain. Paleo, numéro spécial, p. 49-56.

DELPECH F., LENOIR M. 1996 - Éléments d'interprétation sur l'occupation magdalénienne de la bordure nord du massif pyrénéen. In : Pyrénées préhistoriques arts et sociétés. Actes du 118e congrès national des sociétés historiques et scientifiques, DELPORTE H. et CLOTTES J. (Dirs). Paris, éditions du C.T.H.S., p. 217-223.

DELPECH F., PRAT F. 1980 - Les grands mammifères pléistocènes du Sud-ouest de la France. In Problèmes de stratigraphie quaternaire en France et dans les pays limitrophes. Bulletin de l'Association Française pour l'Étude du Quaternaire, supplément n 1, p. 268-297.

Faunmap working Group : GRAHAM R.W., LUNDELIUS Jr E.L., GRAHAM M.A., SCHROEDER E.K., TOOMEY III R.S., ANDERSON E., BARNOSKY A.D., BURNS J.A., CHURCHER C.S., GRAYSON D.K., GUTHRIE R.D., HARINGTON C.R., JEFFERSON G.T., MARTIN L.D., MCDONALD H.G., MORLAN R.E., SEMKEN Jr H.A., WEBB S.D., WERDELIN L., WILSON M.C 1996 Spatial Response of Mammals to Late Quaternary Environment Fluctuations, Science, Reprint Series, 272, p. 1601-1606.

FEYFANT L., COCHARD D., MALLYE J.-B. 2015 - Exploitation du cheval au Magdalénien supérieur dans le Sud-ouest de la France. Le cas de l'abri Faustin (Cessac, Gironde). Bulletin de la Société préhistorique française, tome 112, $\mathrm{n}^{\circ} 4$, octobre-décembre 2015, p. 693-716.

FOSSE P., MADELEINE S., OBERLIN C., CRETIN C., BONNETJACQUEMENT P. 2017 - Un lion des cavernes (Panthera (Leo) spelaea) exploité au Dryas récent: les données du gisement du Peyrat (Saint-Rabier, Dordogne, France), Paleo 28, p. 91-115.

GILBERT A.1984 - Contribution à l'étude des faunes de la fin des temps glaciaires et du début des temps postglaciaires. Thèse d'Université, mention Sciences $n^{\circ} 157$, Université Bordeaux I, 322 p. ronéot.

GRAYSON D.K., DELPECH F. 2003 - Ungulates and the Middle-to-Upper Paleolithic transition at Grotte XVI (Dordogne, France). Journal of Archaeological Science 30, p. $1633-1648$

GRIGGO Ch. 1996 - Établissement de courbes climatiques quantifiées à partir des communautés animales pléistocènes suivi d'une application aux gisements de l'abri Suard (Charente) et la grotte de Bois-Ragot (Vienne). Paleo 8, p. 80-97.
GUADELLI J.-L. 1996 - Les Cerfs du Würm ancien en Aquitaine. Paleo 8 p. 99-108.

GUADELLI J.-L. 2011 - Les associations de grands mammifères de la séquence du Pléistocène supérieur de CombeGrenal (Dordogne, France) et la biochronologie des stades isotopiques 5 à 3. In DELPECH F. et JAUBERT J. (dirs) François Bordes et la Préhistoire. Paris éditions du C.T.H.S., Documents préhistoriques 29, p. 199 - 219.

KUNTZ D. 2011 - Ostéométrie et migration(s) du renne (Rangifer tarandus) dans le Sud-Ouest de la France au cours du dernier Pléniglaciaire et du Tardiglaciaire (2150013000 cal. BP) (Ph.D. thesis). Université de Toulouse 2-Le Mirail, France.

IVERSEN J. 1954 - The Lateglacial flora of Denmark and its relation to climate and soil. Danmark Geologiske Undersoglle 2, 80, p. 87-119.

JANSSENS L., PERRI A., CROMBÉ Ph., VAN DONGEN S., LAWLER D. 2019 - An evaluation of classical morphologic and morphometric parameters reported to distinguish wolves and dogs. Journal of Archeological Science 23, p. 501-533.

JONES E.L. 2009 - Climate change, patch choice, and intensification at Pont d'Ambon (Dordogne, France) during the Younger Dryas. Quaternary Research 72, p. 371-376.

KLEIN R.G., SCOTT K. 1989 - Glacial/Interglacial Size Variation in Fossil Spotted Hyenas (Crocuta crocuta) from Britain. Quaternary Research, 32, p. 88-95.

LANGLAIS M. 2018 - Le Magdalénien supérieur : une technologie de pointes. In AVERBOUH A., BONNETJACQUEMENT P., CLEYET-MERLE J.-J. (dirs.) - L'Aquitaine à la fin des temps glaciaires. Les sociétés de la transition du Paléolithique final au début du Mésolithique dans l'espace Nord aquitain. Paleo, numéro spécial, p. 97-108.

LANGLAIS M., NAUDINOT N., PERESANI M. (dirs.) 2014 - Les groupes culturels de la transition Pléistocène-Holocène entre Atlantique et Adriatique. Séance de la Société préhistorique française, volume 3, $246 \mathrm{p}$.

LAROULANDIE V., COSTAMAGNO S., LANGLAIS M., PÉTILLON J.-M. 2017 - L'œuf ou la poule? Retour sur le projet Magdatis «Le Magdalénien de la façade atlantique face aux changements environnementaux ». Quaternaire, volume $28, n^{\circ} 2$, p. 277-283.

LE GALL O. 1984 - L'ichtyofaune d'eau douce dans les sites préhistoriques. Ostéologie, Paléoécologie, Palethnologie. Cahiers du Quaternaire, $n^{\circ}$ 8, éditions du CNRS, 196 p.

MALLYE J.-B., KUNTZ D., LANGLAIS M., BOUDADI-MALIGNE M., BARSHAY-SZMIDT C., COSTAMAGNO S., PÉTILLON J.-M., GOURICHON L., LAROULANDIE V. 2018 - Trente ans après, que reste-t-il du modèle d'azilianisation proposé au Morin par F. Bordes et D. de Sonneville-Bordes ? In : AVERBOUH A., BONNET-JACQUEMENT P., CLEYET-MERLE J.-J. (dirs.) - L'Aquitaine à la fin des temps glaciaires. Les sociétés de la transition du Paléolithique final au début du Mésolithique dans l'espace Nord aquitain. Paleo, numéro spécial, p. 153-166. 
MANGERUD J., ANDERSEN S.T., BERGLUND B.E., DONNER J.J. 1974 - Quaternary stratigraphy of Norden, a proposal for terminology and classification. Boreas, vol. 3, p. 109-128.

MARQUET J.C. 1989 - Paléoenvironnement et chronologie des sites du domaine atlantique français d'âge pléistocène moyen et supérieur d'après l'étude des rongeurs. Thèse de Doctorat d'État es Sciences naturelles. Université de Bourgogne, 637 p. ronéot.

OBERLIN C. 2007 - L'archéologie et la datation par spectrométrie de masse couplée à un accélérateur (SMA). Les nouvelles de l'archéologie, 107, p. 47-50.

PRAT F., SUIRE C. 1971 - Remarques sur les cerfs contemporains des deux premiers stades würmiens. Bulletin de la Société préhistorique française, Volume 68, 3 : 75-79, 1 fig., 4 tabl.

RAMADE François, 1984 - Éléments d'écologie fondamentale. Paris, McGraw-Hill, 397 p.

RASMUSSEN S.O., ANDERSEN K.K., SVENSSON A.M., STEFFENSEN J.P., VINTHER B.M., CLAUSEN H.B., SIGGAARD-ANDERSEN M.L., JOHNSEN S.J., LARSEN L.B., DAHL-JENSEN D., BIGLER M., RÖTHLISGERGER R., FISHER H., GOTO-AZUMA K., HANSSON M.E., RUTH U. 2006 - A new Greenland ice core chronology for the last glacial termination. Journal of Geophysical Research, vol. 111, D06102, doi:10.1029/2005JD006079

RASMUSSEN S.O., BIGLER M., BLOCKLEY S.P., BLUNIER Th., BUCHARDT S.L., CLAUSEN H.B., CVIJANOVIC I., DAHLJENSEN D., JOHNSEN S.J., FISHER H., GKINIS V., GUILLEVIC M., HOEK W.Z., LOWE J.J., PEDRO J.B., POPP T., SEIERSTAD I.K., STEFFENSEN J.P., SVENSSON A.M., VALLELONGA P., VINTHER B.M., WALKER M., WHEATLEY J.J., WINSTRUP M. 2014 - A stratigraphy framework for abrupt climatic changes during the last Glacial period based on three synchronized Greenland ice-core records : refining and extending the INTIMATE event stratigraphy. Quaternary Science Reviews, 106, p. 14-28.
RICHARD H., BÉGEOT C., GAUTHIER É., RUFFALDI P. 2000 Évolution du couvert végétal du Tardiglaciaire et du début de l'Holocène sur la chaîne jurassienne : nouveaux résultats. In : Les derniers chasseurs-cueilleurs d'Europe occidentale, actes du colloque international de Besançon, octobre 1998. Besançon, Presses Universitaires Franccomtoises, p. 29 à 36 (Annales Littéraires, 699; Série « Environnement, sociétés et archéologie ».

SUIRE C. 1969 - Contribution à l'étude du genre Canis d'après des vestiges recueillis dans quelques gisements pléistocènes du Sud-Ouest de la France, Thèse de ze cycle, Faculté des Sciences de l'Université de Bordeaux, n 638 , 182 p. ronéot,

SZMIDT C., LAROULANDIE V., DACHARY M., LANGLAIS M., COSTAMAGNO S. 2009 - Harfang, Renne et Cerf : nouvelles dates ${ }^{14} \mathrm{C}$ par SMA du Magdalénien supérieur du Bassin aquitain au Morin (Gironde) et Bourrouilla (PyrénéesAtlantiques). Bulletin de la Société préhistorique française, tome $106, n^{\circ} 3$, p. 583-601.

TEXIER J.-P., DELPECH F. 2011 - Les paléoenvironnements de la Grande Plaine Européenne au Tardiglaciaire. In Mille et une femmes de la fin des temps glaciaires. Musée National de Préhistoire, les Eyzies de Tayac, 17 juin-19 septembre 2011. Éditions de la réunion des musées nationaux - Grand Palais, Paris 2011, p. 17 à 29. 
